ज્પ FRANÇAISE

$>\mathrm{DE}$

띠 PÉDAGOGIE

\section{Revue française de pédagogie}

Recherches en éducation

172 | juillet-septembre 2010

La pédagogie universitaire : un courant en plein développement

\title{
Étude de trois facteurs clés pour comprendre la persévérance à l'université
}

Study of three key-elements to better understand why so many students drop out of university

Estudio de tres factores claves para comprender la perseverancia en la universidad

Untersuchung dreir Schlüsselfaktoren, um die Ausdauer an der Universität zu verstehen

Julia Schmitz, Mariane Frenay, Sandrine Neuville, Gentiane Boudrenghien, Vincent Wertz, Bernadette Noël et Jacquelynne Eccles

\section{OpenEdition}

Édition électronique

URL : http://journals.openedition.org/rfp/2217

DOI : $10.4000 / \mathrm{rfp} .2217$

ISSN : 2105-2913

Éditeur

ENS Éditions

Édition imprimée

Date de publication : 15 juillet 2010

Pagination : 43-61

ISBN : 978-2-7342-1188-4

ISSN : 0556-7807

\section{Référence électronique}

Julia Schmitz, Mariane Frenay, Sandrine Neuville, Gentiane Boudrenghien, Vincent Wertz, Bernadette

Noël et Jacquelynne Eccles, «Étude de trois facteurs clés pour comprendre la persévérance à

l'université », Revue française de pédagogie [En ligne], 172 | juillet-septembre 2010, mis en ligne le 01

décembre 2014, consulté le 30 avril 2019. URL : http://journals.openedition.org/rfp/2217 ; DOI :

$10.4000 /$ rfp. 2217 


\section{Étude de trois facteurs clés pour comprendre la persévérance à l'université}

\section{Julia Schmitz, Mariane Frenay, Sandrine Neuville, Gentiane Boudrenghien, Vincent Wertz, Bernadette Noël et Jacquelynne Eccles}

La première année à l'université est marquée par un taux de réussite et de persévérance assez faible. Pour expliquer ce phénomène, on a d'abord pris en compte les caractéristiques sociodémographiques et le passé scolaire des étudiants. Mais, depuis une trentaine d'années, d'autres dimensions ont été étudiées en se basant sur des courants théoriques distincts. D'une part, les modèles éducationnels (Tinto, 1975, 1997 ; Bean, 1980) soulignent à la fois l'importance des expériences sociales et académiques vécues lors de la transition à l'université et l'importance de l'ajustement entre les caractéristiques individuelles et institutionnelles; d'autre part, les modèles motivationnels (Wigfield \& Eccles, 2000 ; Eccles \& Wigfield, 2002 ; Kahn \& Nauta, 2001) se focalisent sur les attributs personnels des étudiants (par exemple le sentiment de compétence, les représentations des buts, etc.). En validant le modèle de Tinto dans le contexte éducationnel belge et en analysant le rôle complémentaire du sentiment d'efficacité personnelle, nous tentons ici de mettre en évidence l'apport de ces différents courants théoriques dans la compréhension des facteurs de persévérance. Cette contribution s'intéresse particulièrement à l'intention de persévérance, mesurée dès les premières semaines passées à l'université, car elle constitue l'un des facteurs majeurs dans la persévérance effective et est positivement associée à la réussite finale. Nos résultats démontrent que la persévérance et la réussite, loin d'être déterminées exclusivement par le bagage d'entrée de l'étudiant, sont significativement influencées par des variables individuelles et des variables contextuelles, ainsi que par l'interaction entre les deux. Cet article met ainsi en lumière l'importance du contexte éducatif et le rôle que peuvent jouer les acteurs du terrain. Ces derniers peuvent soutenir la persévérance et la réussite des étudiants, non seulement par la mise en œuvre de dispositifs pédagogiques adaptés (misant sur les relations entre pairs, l'apprentissage coopératif, l'utilité et l'intérêt des activités), mais aussi par leurs interventions auprès des étudiants pour soutenir leur sentiment d'efficacité personnelle et la construction de leur projet de formation.

Descripteurs (TESE) : attitude envers l'école, participation des étudiants, intégration sociale, transition de l'enseignement secondaire supérieur à l'enseignement supérieur, confiance en soi, progrès scolaire. 


\section{INTRODUCTION}

La transition entre l'enseignement secondaire et l'enseignement supérieur est souvent perçue comme une phase stressante par les étudiants (Shaver, Furman \& Buhrmester, 1985), notamment par ceux qui quittent le foyer familial. Cette transition implique de nombreux changements dans leur vie, tels qu'une charge de travail plus importante, la pression exercée par la réussite des études, l'éloignement de la famille et des amis et la création d'un nouveau réseau social (Paul \& Brier, 2001; Oswald \& Clark, 2003 ; Parker, Summerfeld, Hogan et al., 2004). Bernier, Larose et Whipple (2005) mentionnent également que les étudiants doivent rapidement assumer de nouvelles "responsabilités d'adulte» et vivre sans la surveillance immédiate de leurs parents.

L'adaptation à ces différents changements pourrait expliquer notamment pourquoi les taux d'échec et d'abandon sont élevés en première année d'université (Bentein, Frenay, Verwaerde et al., 2003 ; Romainville, 2000). Les chiffres fournis par la banque de données du conseil des recteurs de la Communauté française de Belgique (CREF) indiquent qu'en moyenne, pour les cinq dernières années, environ $60 \%$ des étudiants belges entrant à l'université pour la première fois n'ont pas réussi leur première année. Parmi ceux-ci, $20 \%$ ont abandonné leurs études. Toutefois, comme le souligne Romainville (2005), ces chiffres doivent être replacés dans le contexte éducatif belge qui offre, à l'exception des études d'ingénieur civil, un libre accès aux études universitaires. Et puisque les étudiants peuvent choisir librement une filière d'études dans l'enseignement supérieur - la seule condition nécessaire étant d'avoir obtenu le certificat d'enseignement secondaire supérieur (CESS), délivré par le conseil de classe de leurs enseignants de sixième année du secondaire (1) -, la première année à l'université peut être considérée comme une année d'orientation et de sélection. Un tel contexte de libre accès se distingue fortement de la plupart des autres contextes d'enseignement supérieur, dans lesquels la sélection se fait avant même l'entrée dans l'enseignement supérieur.

Les déterminants de la réussite et de l'abandon dans l'enseignement supérieur ont préoccupé les chercheurs depuis des décennies (voir notamment Cooke, Bewick, Barkham et al., 2006 ; Pascarella \& Terenzini, 2005). Dans leur livre How college affects students, Pascarella et Terenzini (1991) ont identifié plus de 3000 de ces recherches, menées dans les années soixante-dix et quatre-vingt. Ces auteurs suggèrent que plusieurs théories tentent d'expliquer l'abandon et la persévérance dans les études universitaires. Les modèles les plus courants sont des modèles éducationnels et des modèles motivationnels (Robbins, Lauver, Le et al., 2004). Les modèles éducationnels (Tinto, 1975 ; Bean, 1980 ; Cabrera, Castaneda, Nora et al., 1992 ; Cabrera, Nora \& Castaneda, 1993) soulignent l'importance des expériences institutionnelles et de l'intégration de l'étudiant : ce sont les interactions entre l'individu et l'environnement institutionnel qui déterminent la décision d'abandon ou de persévérance. Les théories motivationnelles, qui ont plus récemment émergé en tant que modèle explicatif de la persévérance, se focalisent davantage sur les caractéristiques de la personnalité, la motivation et les styles d'apprentissage.

Dans cet article, nous étudions les facteurs de persévérance en première année à l'université. Nous nous demandons pourquoi certains étudiants s'adaptent aux changements dont ils font l'expérience à l'entrée à l'université, alors que d'autres ne parviennent pas à surmonter les difficultés et abandonnent plus facilement. Et, plus précisément, nous cherchons à apprécier le poids des facteurs individuels dans la décision de persévérer, pour comprendre dans quelle mesure l'intégration de l'étudiant et son attachement à l'université comptent dans cette décision. L'objectif de cette contribution est double. Premièrement, nous tentons de valider empiriquement le modèle éducationnel le plus répandu dans l'étude de la persévérance, le modèle de Tinto (1975). Concernant les étudiants belges francophones, nous essayons de voir si les facteurs clés avancés par Tinto sont déterminants dans la décision de persévérance. Deuxièmement, face aux critiques du modèle de Tinto (1975), et en raison de l'apport complémentaire des théories motivationnelles dans l'étude de la persévérance, nous testerons un modèle alternatif, qui inclut le sentiment d'efficacité personnelle au sein du modèle de Tinto. En confrontant ces deux modèles, nous espérons ainsi améliorer notre compréhension du phénomène de l'abandon et de la persévérance des étudiants universitaires.

\section{PRÉSENTATION DES DIFFÉRENTS MODÈLES}

\section{Les modèles éducationnels}

\section{Le modèle d'intégration de Tinto}

Vincent Tinto a exposé en 1975 un modèle interactionniste fondé sur les concepts d'intégration et 
d'appartenance à la communauté universitaire. Cet auteur conçoit la persévérance à l'université comme un processus longitudinal d'interactions entre l'étudiant et les systèmes académique et social de l'institution dans lesquels il s'inscrit. L'abandon résulterait d'un manque de congruence entre les caractéristiques de l'étudiant et celles de l'institution choisie (Cabrera, Castaneda, Nora et al., 1992; Kahn \& Nauta, 2001), et aussi d'un manque d'intégration de l'étudiant dans l'institution. Ce modèle initial de Tinto et ses développements (Tinto, 1987, 1993) figurent parmi ceux qui sont les plus connus et les plus utilisés dans l'étude de la persévérance et de l'abandon à l'université (Braxton, 2000; Kahn \& Nauta, 2001 ; Pascarella \& Terenzini, 2005). Braxton (2000), après avoir trouvé plus de 400 citations de ce modèle en 1994, lui accorde un statut presque paradigmatique - une estimation plus récente corrige l'estimation à 775 citations (Braxton \& Hirschy, 2005).

Dans ce modèle, on considère que l'étudiant entre à l'université avec tout un bagage, fait de ses caractéristiques personnelles, familiales et scolaires, ainsi que des buts qu'il poursuit (goal commitment (2)), ici essentiellement celui d'obtenir un diplôme, et de son engagement institutionnel (institutional commitment). Le modèle postule que ces caractéristiques influenceront les expériences vécues par les étudiants au sein des systèmes académique et social de l'université, et détermineront en conséquence leur degré d'intégration académique et d'intégration sociale. L'intégration académique est mesurée, d'une part, en termes de performance scolaire et de développement intellectuel de l'étudiant et, d'autre part, par l'identification de l'étudiant aux normes du système académique, c'est-à-dire par le degré de congruence entre les valeurs et objectifs de l'étudiant et ceux de l'institution. L'intégration sociale est quant à elle définie par l'interaction entre l'étudiant et les intervenants du système (professeurs, pairs, etc.) et est mesurée par le degré de congruence entre l'étudiant et l'environnement social de l'institution (principalement, à travers les interactions sociales qui se nouent avec le groupe de pairs et les interactions informelles avec les enseignants, dans les dispositifs pédagogiques coopératifs notamment). De ce fait, les expériences négatives sont celles qui réduisent le degré d'intégration, qui créent une distance entre l'individu et le système académique et social de l'institution et qui favorisent un désengagement. Tinto insiste sur le fait que la qualité des expériences vécues au sein des systèmes académique et social (mesurée par le degré d'intégration académique et sociale) modifie les engagements initiaux des étudiants (le goal commitment et l'institutional commitment), et ce sont ces engagements ultérieurs qui détermineront soit la persévérance, soit l'abandon. On peut donc voir que le concept d'intégration est le pivot du modèle de Tinto (1975).

Des études ayant examiné le modèle de Tinto (1975, 1987) dans son entièreté (Pascarella \& Terenzini, 1980, 1983 ; Cabrera, Castaneda, Nora et al., 1992 ; Cabrera, Nora \& Castaneda, 1993), ainsi que la métaanalyse réalisée par Braxton, Sullivan et Johnson (1997), soutiennent une grande partie des propositions faites par Tinto. Dans ce dernier cas, les auteurs ont examiné plus de 300 études et ont tenté de vérifier si les quinze hypothèses d'interactions suggérées par Tinto dans son modèle initial (1975, voir aussi figure 1) obtenaient une validation empirique. Six de ces hypothèses sont confirmées par une grande majorité des études prises en compte et concernent l'impact des caractéristiques individuelles sur l'engagement institutionnel initial, la modification des engagements (le goal commitment et l'institutional commitment) sous l'influence des expériences à l'université et la qualité de l'intégration de l'étudiant, l'impact de l'intégration sociale sur l'engagement institutionnel ultérieur, l'impact de l'engagement institutionnel ultérieur sur la décision de persévérance, et enfin les interactions entre les intégrations académique et sociale (Cabrera, Castaneda, Nora et al., 1992).

Cette métaanalyse et d'autres études (Pascarella \& Terenzini, 1983 ; Braxton, Milem \& Sullivan, 2000 ; Schmitz, Frenay, Neuville et al., 2007 ; Schmitz, 2009) soulignent l'importance de l'intégration sociale dans le processus de persévérance, particulièrement en raison de son influence sur l'engagement institutionnel ultérieur. Certaines études montrent également des effets directs de l'intégration sociale sur l'intention de persévérance (Berger \& Milem, 1999) et seules quelques rares études ne parviennent pas à montrer un lien significatif sur l'intention de persévérance (Torres \& Solberg, 2001). Cependant certaines des affirmations majeures du modèle de Tinto ne sont pas validées empiriquement. C'est le cas du rôle clé de l'intégration académique vis-à-vis des buts poursuivis, qui n'est confirmé que par cinq études sur douze (notamment celles de Pascarella \& Terenzini, 1983 ; Pascarella, Duby \& Iverson, 1983). La viabilité même du concept d'intégration académique, à propos des études sur la persévérance, a été mise en question par Braxton et Lien (2000). De même, il apparaît que les sources de l'intégration sociale ne sont pas clairement identifiées (Braxton, Milem \& Sullivan, 2000). 


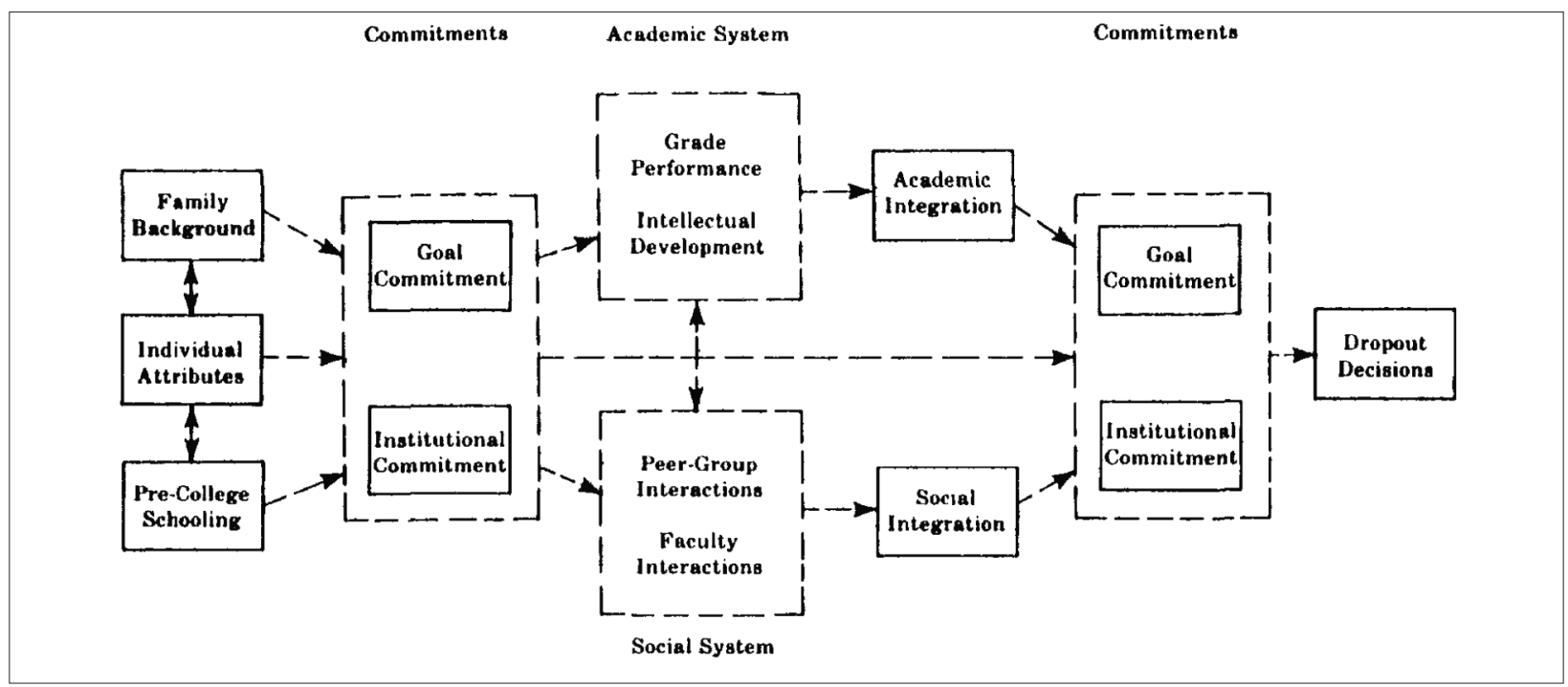

\section{Le modèle intégratif de Cabrera}

En 1992 et 1993, Cabrera et ses différents collègues (Cabrera, Castaneda, Nora et al., 1992 ; Cabrera, Nora \& Castaneda, 1993) ont tenté de comparer le modèle de Tinto $(1975,1987)$ avec un autre modèle, "classique ", de persévérance: le modèle d'abandon (3) de Bean (1980, 1983). Au niveau conceptuel, le modèle de Bean présente des similitudes avec le modèle de Tinto, telles que l'importance d'une congruence ou d'un ajustement entre les caractéristiques de l'étudiant et celles de l'institution (l'institutional fit) et la prise en compte des caractéristiques individuelles (bagage familial, passé scolaire, etc.) à l'entrée à l'université. Le modèle initial de Bean (1980) et ses développements ultérieurs (Bean, 1983 ; Bean \& Metzner, 1985) se distinguent du modèle de Tinto en relevant avant tout l'impact des facteurs environnementaux (encouragement de la famille et des amis, opportunités de changement d'université, difficultés financières) et celui des intentions comportementales (intention de persévérance) dans l'étude de la persévérance (Bean \& Vesper, 1990 ; Cabrera, Castaneda, Nora et al., 1992 ; Cabrera, Nora \& Castaneda, 1993). Selon Bean, les expériences sociales et académiques influencent la perception par l'étudiant de différentes facettes de la vie universitaire, comme la qualité de l'établissement, la satisfaction des cours, les relations avec les amis et enseignants, etc. À leur tour, les perceptions positives mènent à une perception de congruence de l'étudiant avec l'institution, qui déterminera en grande partie les intentions de persévérance.

Dans leur étude comparative, Cabrera et ses collègues (1992) soulignent l'importance de la congruence entre l'étudiant et l'institution et de l'intention de persévérer: "Les résultats des deux modèles indiquent que la persévérance à l'université résulte d'un processus d'interactions entre les facteurs personnels et institutionnels, et que l'intention de persévérance est déterminée par une congruence entre l'étudiant et l'institution " (Cabrera, Castaneda, Nora et al., 1992, p. 158). Ces auteurs accordent au modèle de Tinto $(1975,1987)$ une puissance prédictive plus importante que le modèle de Bean (1980) et ses développements de la persévérance. Partant de cette analyse, ces chercheurs proposent en 1993 un modèle intégratif de ces deux modèles théoriques (cf. figure 2).

Les résultats des études qu'ils ont menées auprès de 2456 étudiants (4) entrant à l'université confirment les idées principales du modèle de Tinto, notamment l'impact de l'intégration sociale de l'étudiant sur l'engagement institutionnel ultérieur et l'impact de l'intégration académique sur les engagements (le goal commitment et l'institutional commitment) ultérieurs. Pour mesurer ces impacts, on utilisera par la suite le coefficient $\beta$, qui est un estimateur standardisé des paramètres relatifs aux relations entre les différentes variables, indiquant la force de la relation entre les deux variables associées (Jöreskog \& Sörbom, 1993). Quand ce 


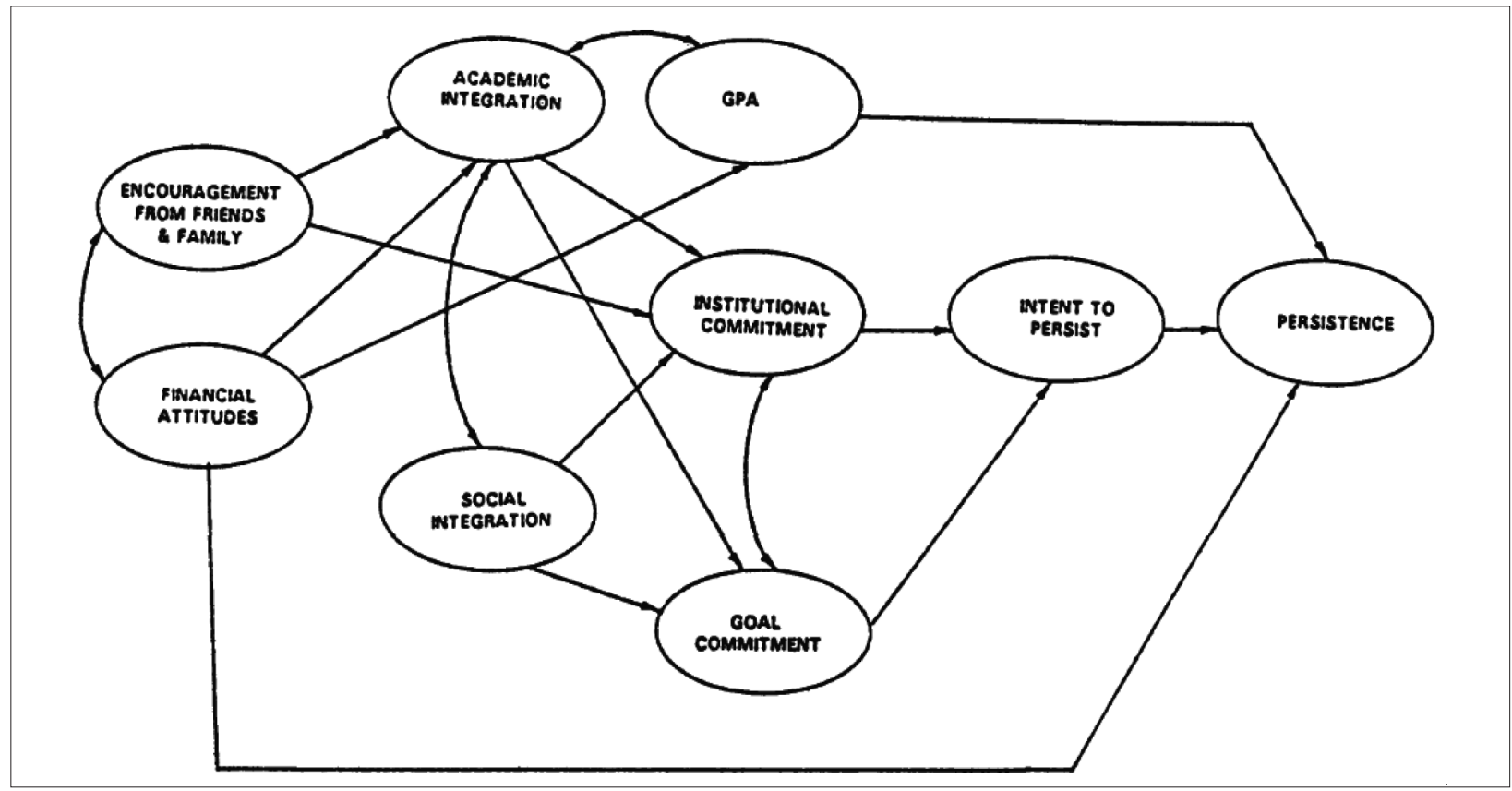

Note : le Grade point average (ou GPA) est ici une mesure de performance académique des étudiants.

coefficient est inférieur à 0,05 , les relations ne sont pas significatives. En ce qui concerne l'intention de persévérance, les résultats indiquent qu'elle est largement déterminée par l'engagement institutionnel $(\beta=0,51)$ et dans une moindre mesure par les buts poursuivis $(\beta=0,28)$. Conformément au modèle de Bean et Metzner (1985), l'intention de persévérance influence fortement la persévérance effective $(\beta=0,48)$, tout comme la performance académique $(\beta=0,45)$. Par ailleurs, les résultats du modèle intégratif de Cabrera (Cabrera, Nora \& Castaneda, 1993) mettent en évidence l'impact d'un facteur environnemental mis en avant par Bean et Metzner (1985), celui du soutien familial et des proches, sur les intégrations académique $(\beta=0,32)$ et sociale $(\beta=0,35)$ des étudiants (5).

\section{Les modèles motivationnels}

\section{Présentation de quelques modèles} motivationnels

Au sein de la littérature de recherche en psychologie, nous pouvons constater que les modèles motivationnels constituent une alternative intéressante dans la prédiction de la réussite académique et de la persévérance (Robbins, Lauver, Le et al., 2004). Ces modèles mettent l'accent sur la prédiction des comportements ou des changements de comportement en fonction des facteurs individuels tels que la motivation, la valeur accordée à la tâche, le sentiment d'efficacité personnelle, les buts poursuivis. Le sentiment d'efficacité personnelle est défini comme la perception qu'a la personne de ses capacités à mettre en œuvre les activités nécessaires à la réalisation d'une tâche donnée. Selon Robbins et ses collègues (2004), le modèle expectancy-value (Wigfield \& Eccles, 2000 ; Eccles \& Wigfield, 2002) est particulièrement pertinent pour comprendre la persévérance et l'engagement des étudiants. Cette théorie propose que les perceptions des étudiants concernant leur probabilité de réussite d'une tâche donnée (expectancy perceptions), ainsi que leurs perceptions de ce que cette tâche peut leur apporter (value perceptions), sont les déterminants les plus immédiats des comportements liés à l'apprentissage, tels que le choix du sujet de s'engager et/ou de persévérer dans la réalisation d'une tâche, les stratégies d'apprentissage que le sujet va mettre en œuvre pour appréhender cette tâche et, enfin, sa performance à celle-ci. Des études récentes réalisées à Louvain-la-Neuve montrent que ce cadre théorique s'applique également à la prédiction de l'intention de persévérer (Neuville, Frenay, 
Schmitz et al., 2007 ; Neuville \& Frenay, 2010), tout autant qu'à la persévérance effective des étudiants universitaires (Neuville \& Frenay, 2010). De plus, en ce qui concerne la prédiction de la réussite académique, ces auteurs ont constaté une efficacité prédictive plus grande du modèle d'expectancy-value par rapport à celui de Tinto (1975) et un impact important du sentiment d'efficacité personnelle, mesuré au cours de l'année, sur l'intention de persévérer (Neuville, Frenay, Schmitz et al., 2007).

Ces modèles motivationnels, notamment ceux centrés principalement sur le sentiment d'efficacité personnelle, s'avèrent donc utiles dans la prédiction de la persévérance (Kahn \& Nauta, 2001; Robbins, Lauver, Le et al., 2004 ; Galand \& Frenay, 2005 ; Neuville \& Frenay, 2010).

\section{La théorie du sentiment d'efficacité personnelle}

Depuis l'introduction de la théorie de l'apprentissage social par Bandura (1997), le concept d'efficacité personnelle a joué un rôle central dans les tentatives de compréhension et de prédiction du comportement humain. Quatre sources sont à l'origine de ce sentiment d'efficacité personnelle: les expériences actives de maîtrise, notamment dans les tâches scolaires ou académiques (performances antérieures, succès, échecs), les expériences vicariantes (modelage, comparaison sociale), la persuasion verbale (feedback évaluatifs, encouragements, avis de personnes signifiantes) et les états physiologiques et émotionnels. Au centre de cette théorie, se trouve l'hypothèse selon laquelle les croyances d'efficacité personnelle aident les gens à déterminer le type d'activités à poursuivre, les efforts qu'ils consacrent pour les activités poursuivies et la persévérance face aux obstacles. Bandura (1997) affirme que le sentiment d'efficacité personnelle affecte les résultats à l'université en agissant favorablement sur la motivation des étudiants et leur persévérance face aux exigences académiques et aux difficultés rencontrées.

Multon, Brown et Lent (1991) ont réalisé une métaanalyse très vaste concernant le lien entre les croyances d'efficacité personnelle (elles-mêmes en lien avec des tâches académiques), la performance et la persévérance. Les auteurs constatent une supériorité du sentiment d'efficacité personnelle académique, faisant référence à la confiance dans la capacité à réaliser des tâches académiques avec succès, telles que la préparation d'un examen ou la rédaction d'un travail, par rapport à des mesures moins spécifiques. Les résultats de cette métaanalyse suggèrent qu'entre
$11 \%$ et $14 \%$ de la variance liée à la performance scolaire et à la persévérance peut être expliquée par les croyances d'efficacité personnelle de l'individu. Depuis l'étude de Multon et ses collègues (1991), de nombreuses recherches ont montré que le sentiment d'efficacité personnelle est positivement associé à la performance à l'université (Gore, Leuwerke \& Turley, 2006 ; Robbins, Lauver, Le et al., 2004 ; Chemers, Hu \& Garcia, 2001; Bong, 2001) et à la persévérance universitaire (Robbins, Lauver, Le et al., 2004 ; Torres \& Solberg, 2001 ; Karpanty, 1998).

La sociocognitive career theory (6) a également intégré le sentiment d'efficacité personnelle dans son explication théorique de la façon dont les étudiants construisent leurs choix vocationnels et professionnels (Betz \& Hackett, 1981), en proposant le concept de "career-decision making self-efficacy", qu'ils définissent comme la confiance perçue des étudiants dans leur capacité à planifier et réaliser les tâches étroitement liées à leur développement professionnel au sein du milieu éducatif (notamment rassembler les informations, planifier et expliciter les objectifs, voir par exemple Betz \& Hackett, 1981 ; Peterson, 1993). Cette théorie suggère qu'outre les aptitudes scolaires ou la performance scolaire antérieure, la persévérance à l'université serait influencée par la confiance qu'a un étudiant dans ses capacités académiques (le sentiment d'efficacité personnelle), par ses attentes en termes de résultats académiques, ainsi que par sa détermination à réussir et à obtenir le diplôme (Kahn \& Nauta, 2001). Contrairement aux modèles précédents, Kahn et Nauta (2001) n'ont pas pu mettre en évidence le rôle de ces trois variables dans leurs recherches, variables qui ont été mesurées au cours du premier semestre. Cependant les résultats des mesures effectuées durant le second semestre à l'université indiquent que le sentiment d'efficacité personnelle et la détermination de l'étudiant ont un impact significatif sur la persévérance. Ces auteurs considèrent cette théorie comme une théorie cognitivo-motivationnelle présentant un changement important par rapport aux précédents efforts pour comprendre la persévérance, à savoir ceux de Tinto (1975) et de Bean (1980). Les auteurs insistent sur la pertinence d'inclure des facteurs motivationnels dans les études focalisées sur la persévérance à l'université.

\section{Aperçu de quelques études intégratives}

Certaines études ont tenté de réviser le modèle de Tinto $(1975,1993)$ en y incluant des attributs motiva- 
tionnels (Peterson, 1993). D'autres études ont combiné quelques aspects clés issus de différents modèles théoriques (notamment Torres \& Solberg, 2001) dans l'étude de la persévérance. Trois études retiennent particulièrement notre attention, car elles soulignent la complexité du phénomène de persévérance et l'importance de combiner les apports des théories éducationnelles et motivationnelles. Peterson (1993) a été l'un des premiers à inclure le sentiment d'efficacité personnelle dans le modèle de Tinto (1987). Son but était d'examiner l'impact du sentiment d'efficacité personnelle lié à la carrière professionnelle (ou career-decision making self-efficacy) sur l'intégration académique, sur l'intégration sociale, ainsi que sur les engagements initiaux. Peterson a réalisé son étude auprès de 418 étudiants inscrits dans un programme universitaire conçu pour des étudiants en difficulté académique. Les résultats de son étude ont montré que l'inclusion du sentiment d'efficacité personnelle lié à la carrière professionnelle influence significativement les engagements initiaux et constitue le meilleur prédicteur $(\beta=0,42)$ de l'intégration académique. En accord avec les propositions de Tinto (1975), les engagements (le goal commitment et l'institutional commitment) initiaux constituent les meilleurs prédicteurs de l'intégration sociale $(\beta=0,35)$, suivis de près par l'impact de ce sentiment d'efficacité personnelle $(\beta=0,25)$.

Inspiré du travail de Peterson (1993), Sandler (2000) a réalisé une étude auprès d'étudiants plus âgés (autour de 24 ans). L'auteur a créé un modèle théorique innovant prédisant la persévérance, en se basant sur le modèle intégratif de Cabrera et ses collègues (1993), et en y ajoutant les concepts du career-decision making self-efficacy (Peterson, 1993), le stress perçu et les difficultés financières. Contrairement à Peterson (1993), Sandler se focalise sur la prédiction de la persévérance et observe des effets positifs du sentiment d'efficacité personnelle sur l'intention de persévérance $(\beta=0,20)$, la persévérance actuelle $(\beta=0,11)$ et la gestion du stress $(\beta=0,16)$. Cependant des effets négatifs du sentiment d'efficacité personnelle sur l'engagement institutionnel $(\beta=-0,11)$, l'intégration académique $(\beta=-0,22)$ et l'intégration sociale $(\beta=-0,14)$ ont été constatés. En accord avec le modèle de Bean $(1980,1983)$ et celui de Cabrera (Cabrera, Nora \& Castaneda, 1993), l'intention de persévérance $(\beta=0,66)$ constitue un prédicteur très puissant de la persévérance effective. D'autres prédicteurs de la persévérance effective sont l'intégration sociale $(\beta=0,15)$, l'intégration académique $(\beta=-0,17)$, l'engagement institutionnel $(\beta=-0,20)$, le soutien de la famille $(\beta=-0,14)$ et la perception des difficultés financières $(\beta=0,12)$.
Enfin Torres et Solberg (2001) ont réalisé une étude prédisant les intentions de persévérance et la santé psychologique et physique de 179 étudiants latinoaméricains sur base de quatre variables théoriques : le sentiment d'efficacité personnelle académique (Bandura, 1986), la perception du stress, la perception du soutien de la famille (Bean, 1983) et l'intégration sociale (Tinto, 1987). De manière générale, les résultats indiquent que les étudiants ayant un sentiment d'efficacité personnelle élevé présentent une meilleure intégration sociale $(\beta=0,50)$, moins de stress académique $(\beta=-0,22)$ et davantage d'intentions de persévérance $(\beta=0,24)$ que ceux qui présentent un sentiment d'efficacité personnelle plus bas. Cette étude montre également que le soutien familial influence positivement le sentiment d'efficacité personnelle $(\beta=0,46)$.

\section{PRÉSENTATION DE NOTRE RECHERCHE}

\section{Objectifs}

Cette revue des écrits scientifiques nous a permis de constater que les modèles éducationnels (Tinto, 1975 ; Bean, 1980 ; Cabrera, Castaneda, Nora et al., 1992 ; Cabrera, Nora \& Castaneda, 1993), ainsi que les théories motivationnelles peuvent expliquer la persévérance ou l'intention de persévérance des étudiants avec un certain succès. Dans un premier temps, on peut observer que les modèles éducationnels constituent des modèles incontournables pour l'étude de la persévérance. De nombreuses études confirment l'importance de l'intégration de l'étudiant et de la congruence entre les caractéristiques de l'étudiant et celles de l'institution. Malgré une supériorité du modèle de Tinto (1975) dans la prédiction de la persévérance, Cabrera et ses collègues (Cabrera, Castaneda, Nora et al., 1992 ; Cabrera, Nora \& Castaneda, 1993) suggèrent que les modèles de Tinto et de Bean (1980) semblent se compléter. La théorie de Tinto fournit des concepts essentiels dans l'étude du processus de persévérance, notamment l'intégration sociale, l'intégration académique, l'engagement institutionnel et les buts poursuivis, alors que le modèle de Bean a permis de mettre en avant l'importance de l'intention de persévérance comme prédicteur de la persévérance effective et le rôle des variables environnementales (notamment le soutien familial).

Les modèles motivationnels constituent un cadre théorique alternatif, focalisé sur les facteurs individuels, pour prédire les comportements de persévé- 
rance et d'abandon. Dans ce contexte, il a été démontré que le sentiment d'efficacité personnelle a une influence importante sur le processus de persévérance et la décision de persévérer des étudiants entrant à l'université (Robbins, Lauver, Le et al., 2004). Vu l'importance de ce facteur, certaines études ont tenté d'intégrer ce concept motivationnel dans le modèle de Tinto (notamment Peterson, 1993) ou dans celui de Cabrera et ses collègues (1993), afin de mieux comprendre son impact sur la décision de persévérance et sur d'autres facteurs clés en lien avec la persévérance (l'engagement institutionnel, l'intégration sociale, l'intégration académique, etc.). En effet, comme le suggèrent plusieurs auteurs, il semble important de tester la pertinence d'autres modèles théoriques dans l'étude de la persévérance académique (Kahn \& Nauta, 2001).

Certaines études intégratives attestent d'un impact du sentiment d'efficacité personnelle sur l'intégration sociale (voir Peterson, 1993 ; Torres \& Solberg, 2001), l'intégration académique, les engagements initiaux et l'intention de persévérance. L'inclusion de ce sentiment au sein du modèle de Tinto (1975), en fournissant une source d'influence de l'intégration sociale, pourrait donc répondre à une critique importante adressée à ce modèle. Cependant des différences significatives apparaissent entre ces études, tant au niveau du type de mesures du sentiment d'efficacité personnelle (comme les sentiments d'efficacité personnelle académique et professionnelle), de la population concernée (étudiants plus âgés que les étudiants traditionnels, étudiants issus des ethnies minoritaires, étudiants peu préparés académiquement) que du degré d'inclusion des facteurs clés du modèle de Tinto (étude ou non de la persévérance, inclusion des engagements initiaux, etc.). Selon Sandler (2000), ces facteurs peuvent éventuellement expliquer des effets négatifs inattendus du sentiment d'efficacité personnelle ou de l'intégration sociale dans les études. Nous rappelons que le modèle de Tinto (1975) a initialement été conçu pour des étudiants considérés comme traditionnels, ce qui n'est pas le cas pour les études intégratives présentées ultérieurement. Une autre explication des effets inattendus peut être liée à l'inclusion de prédicteurs nouveaux. On peut s'interroger sur le pouvoir prédictif de ces différents facteurs quand ils sont combinés simultanément. Certains de ces facteurs se compensent-ils ou s'annulent-ils ?

\section{Méthodologie}

Au vu de cette revue des écrits scientifiques, nous aimerions donc mettre à l'épreuve des faits deux modèles permettant d'expliquer la persévérance à l'université. Le premier modèle permettra ou non de valider empiriquement le modèle d'intégration de Tinto (1975) auprès d'une population d'étudiants belges francophones, qui s'inscrivent pour la première fois à l'université. En cohérence avec les études de Bean et de Cabrera qui soulèvent le rôle primordial des intentions de persévérance, nous cherchons à prédire l'intention de persévérer dans les études, en quantifiant l'impact du passé scolaire des étudiants, des buts qu'ils poursuivent, de leur engagement institutionnel et de leur intégration sociale et académique. Le second modèle reprendra ce premier modèle en y ajoutant, à propos du même échantillon, le sentiment d'efficacité personnelle académique. Nous tenterons ainsi de créer un modèle intégratif qui tienne compte à la fois de l'impact de l'institution et des expériences académiques et sociales, mais qui mette également l'accent sur les facteurs motivationnels susceptibles d'évoluer dans le temps. Les recherches réellement intégratives sont peu nombreuses, et celles qui se proposent de tester des modèles théoriques plus élaborés le sont encore moins (Parmentier, 1994). Nous comparerons le pouvoir prédictif de ces deux modèles, en recourant à des analyses en pistes causales.

Notre échantillon se compose de 2632 étudiants universitaires de première année issus des différentes facultés d'une université belge francophone, principalement résidentielle (c'est-à-dire une université dans laquelle la majorité des étudiants résident sur le campus universitaire). Parmi ceux-ci, 55,6 \% sont des femmes et $44,4 \%$ des hommes. Leur âge moyen est de 18,3 ans (avec un écart type de 1,5) et ils s'inscrivent pour la première fois à l'université. L'ensemble des étudiants a donné son accord pour participer à l'étude ainsi que pour nous permettre d'accéder à leurs résultats académiques. Tous les participants ont été assurés de la confidentialité de leurs réponses. La procédure suivie est la suivante: les données ont été récoltées en trois vagues à l'aide de questionnaires autorapportés. La première récolte a eu lieu au début de l'année académique (septembre 2005, récolte notée T1 par la suite) et avait pour but d'évaluer les données à l'entrée des étudiants à l'université et avant que ceux-ci ne soient influencés par les expériences vécues à l'université. La seconde récolte s'est déroulée environ huit à dix semaines après la première (novembre 2005, récolte notée T2 par la suite); elle poursuivait l'objectif d'appréhender les expériences vécues par les étudiants au sein du système académique et social de l'université, leurs engagements, leur perception du sentiment d'efficacité personnelle et leur intention de persévérance. Dans un troisième temps, 
nous avons récolté les résultats de ces étudiants à la fin de l'année universitaire. En se basant sur notre modèle théorique de référence, la première récolte de données a porté sur le bagage familial de l'étudiant, son passé scolaire, son sentiment d'efficacité personnelle et ses engagements (goal commitment et institutional commitment) à l'entrée à l'université.

Nous avons mesuré les variables impliquées dans nos deux modèles tel qu'indiqué dans la suite. La mesure du sentiment d'efficacité personnelle ne sera introduite que dans l'analyse du second modèle. Le sentiment d'efficacité personnelle par rapport aux tâches académiques liées à la réussite académique et à l'adaptation à l'université a été mesuré par 11 items inspirés du CSEI (College self-efficacy inventory, voir Solberg et al., 1993). Un exemple d'item de cette catégorie est: " Je pense que je serai capable de gérer mon temps. " Ces items ont été testés à deux moments distincts (septembre et novembre). Cette échelle a été validée par Neuville et ses collègues (2007) et présente un alpha de Cronbach (7) de 0,81.

Conformément au modèle de Tinto $(1975,1987)$, la motivation à réussir chez l'étudiant a été mesuré à deux reprises et identifie ici l'importance accordée par l'étudiant à l'obtention du diplôme universitaire (Pascarella \& Terenzini, 1979, 1980 ; Cabrera, Nora \& Castaneda, 1993 ; Sandler, 2000). En septembre, l'engagement a été mesuré à l'aide d'un seul item, le grade (8) visé pour l'année académique : 1 = satisfaction, 2 = distinction, 3 = grande distinction, $4=$ plus grande distinction. En novembre, l'engagement de l'étudiant a été mesuré à l'aide de trois items. À titre d'exemple, voici un des items : " II est important pour moi d'obtenir une bonne note aux examens. " Cette échelle présente un alpha de Cronbach de 0,69.

En accord avec le modèle de Tinto, l'engagement institutionnel a été mesuré à deux reprises. II identifie la satisfaction du choix de l'institution, la perception d'une congruence entre l'étudiant et l'institution (l'institutional fit, voir notamment Pascarella \& Terenzini, 1979, 1980 ; Cabrera, Nora \& Castaneda, 1993) et un sentiment d'attachement à l'université choisie (Sandler, 2000). En septembre, cinq items (dont par exemple : « L'UCL fournit un accompagnement suffisant ") ont mesuré l'engagement initial des étudiants au travers de leurs opinions générales sur l'université choisie. L'alpha de Cronbach était de 0,68 pour cette échelle. En novembre, quatre items (dont par exemple : « Je suis très satisfait(e) d'être à l'UCL ") ont mesuré l'engagement ultérieur sous forme de degré d'attachement à l'université et de satisfaction avec le choix de l'institution. L'alpha de Cronbach était de 0,85 .
Huit items, inspirés des travaux de Cabrera et ses collègues (1993), de Lacante et ses collègues (2001), de Sandler (2000) et de Mannan (2001), ont été utilisés pour mesurer le degré d'engagement et de satisfaction de l'étudiant dans ses relations aux autres étudiants, facette importante de l'intégration sociale définie par Tinto (1975). Cette échelle a été validée par Neuville et ses collègues (2007) et sert à identifier les expériences des étudiants concernant la création de relations interpersonnelles proches et la construction d'amitiés à l'université. Un exemple d'item de cette catégorie est le suivant: « Mes relations avec les autres étudiants se déroulent dans un climat de confiance. " L'alpha de Cronbach pour cette échelle est de 0,83 .

Dix items, inspirés des travaux de Pascarella et Terenzini (1983), de Cabrera et ses collègues (1993) et de Mannan (2001), ont quant à eux mesuré la satisfaction des étudiants par rapport aux expériences académiques. Des exemples d'items de cette catégorie sont les suivants: "Jusqu'à maintenant, je m'en suis bien sorti(e) pour tous les travaux, préparations ", "Je suis très intéressé(e) par le contenu des cours. " Pour la perception qu'ils ont de la préoccupation des enseignants concernant leur développement intellectuel, voici un exemple d'item : "Les enseignants nous encouragent à apprendre de nos erreurs. " Ces deux facettes font partie de l'intégration académique définie par Tinto (1975). L'alpha de Cronbach pour cette échelle est de 0,74.

Nous avons enfin mesuré la persévérance par une mesure proximale, à savoir l'intention de persévérer, en raison de son impact déterminant sur la décision de persévérance (Bean, 1985 ; Cabrera, Castaneda, Nora et al., 1992 ; Cabrera, Nora \& Castaneda, 1993 ; Neuville \& Frenay, 2010). Plus spécifiquement, la satisfaction du choix d'études et le désir de poursuivre dans ce même programme (Torres \& Solberg, 2001; Neuville et al., 2007) ont été mesurés par huit items, dont voici un exemple : « J'ai déjà pensé à abandonner mes études », item «négatif ». L'alpha de Cronbach est ici de 0,78.

\section{ANALYSES ET RÉSULTATS DE NOTRE RECHERCHE}

Pour tester le pouvoir prédictif des deux modèles retenus, nous avons effectué des analyses en pistes causales. Ces analyses ont été menées à l'aide du logiciel Lisrel 8.8. Elles considèrent non seulement le 
modèle à tester dans son ensemble mais, comme celui-ci est traduit comme un système d'équations, chacune de celles-ci permet d'estimer les relations postulées entre les variables (Jöreskog \& Sörbom, 1993). Cette méthode permet de vérifier la pertinence et le sens des relations postulées entre les variables, mais aussi de vérifier l'adéquation de la structure d'ensemble du modèle.

Afin d'évaluer le degré d'adéquation du modèle théorique postulé, nous utilisons quatre indices d'ajustement des données au modèle : le $\chi^{2}$, relativement aux degrés de liberté $\left(\chi^{2} / \mathrm{ddl}\right)$. Cet indice est un indicateur de l'écart entre la matrice de corrélation observée dans les données et la matrice de corrélation dérivée du modèle théorique postulé. En raison d'une trop grande sensibilité de ce test à la taille de l'échantillon, il est conseillé d'examiner la valeur du rapport entre le $\chi^{2}$ et les degrés de liberté du modèle. Une valeur inférieure à 5 indique un bon ajustement du modèle aux données observées. Le comparative fit index (ou $\mathrm{CFI}$ ) compare le modèle hypothétique avec un modèle indépendant qui stipule l'absence de corrélations entre les variables mesurées, alors que l'adjusted goodness-of-fit index (ou AGFI) compare le modèle proposé à une absence de modèle. Cet AGFI tient compte de la parcimonie du modèle et inclut une sanction pour l'addition de paramètres supplémentaires. Le CFI et I'AGFI varient entre 0 (aucune adéquation du modèle proposé) et 1 (adéquation parfaite) et les valeurs égales ou supérieures à 0,90 indique une bonne qualité d'ajustement du modèle. L'indice RMSEA (Root mean square error of approximation) rend compte du degré d'erreur d'approximation dans la population. Une valeur inférieure de cet indice à 0,05 (Hu \& Bentler, 1998) indique un bon ajustement et une valeur comprise entre 0,05 et 0,08 montre un ajustement acceptable. Le standardized root mean square residual (ou sRMR) mesure également la moyenne des résidus du modèle. Les valeurs inférieures à 0,05 indiquent un bon ajustement.

Étant donné le nombre conséquent de variables mesurées dans notre étude, des analyses statistiques préalables à la réalisation des analyses en pistes causales ont été menées afin de défricher les relations entre les variables du modèle. Ces analyses ont consisté en corrélations entre les différentes variables mesurées et en régressions multiples. Les corrélations ont permis d'avoir des informations sur les liens entre les différentes variables prises deux à deux. Les régressions multiples, quant à elles, ont permis de faire un pas de plus et de savoir dans quelle mesure une variable donnée du modèle pouvait être prédite ou expliquée par une ou plusieurs variables de ce même modèle. Ainsi il est apparu que le diplôme de la mère et le genre n'avaient pas un impact significatif sur les engagements initiaux des étudiants, comme l'avait affirmé Tinto (9) (1975). Ces variables ont donc été éliminées des analyses ultérieures en pistes causales, puisque les régressions avaient mis en évidence leur absence de pertinence.

\section{Résultats du premier modèle}

Le premier modèle permet de mettre à l'épreuve la pertinence du modèle initial de Tinto (1975) dans son ensemble. Ce modèle théorique s'adapte-t-il bien aux données que nous avons récoltées? Les indices d'ajustement obtenus par l'analyse en pistes causales nous permettent de répondre positivement à cette question. Les $\operatorname{CFI}(0,98)$ et AGFI $(0,98)$ sont, en effet, tous deux largement supérieurs à 0,90 , le sRMR est bien inférieur à $0,05(0,02)$ et le RMSEA est inférieur à $0,05(0,04)$. De plus, le rapport $\chi^{2} /$ ddl $(54,15 / 11)$ est inférieur à 5 . Les indices de l'ajustement du modèle 1 signifient que le modèle de Tinto fournit une bonne description, une bonne explication de ce qui se passe dans la réalité et de la manière dont les différentes variables interagissent. Étant donné que nous savons désormais que le modèle de Tinto (1975) constitue une grille de lecture adéquate de nos données, portons notre attention sur les liens entre les différentes variables constitutives de ce modèle. La figure 3 présente le modèle 1 ainsi que les coefficients standardisés de régression $(\beta)$, qui permettent d'indiquer le poids des relations entre les variables concernées.

Conformément au modèle de Tinto $(1975,1987)$, l'engagement institutionnel initial affecte les expériences académiques et sociales des étudiants (comme l'intégration académique, $\beta=0,13$ et l'intégration sociale, $\beta=0,06)$. À leur tour, l'intégration sociale et l'intégration académique déterminent l'engagement institutionnel ultérieur $(\beta=0,29$ et $\beta=0,30)$ d'une manière plus importante que l'engagement institutionnel initial $(\beta=0,09)$. Ensuite, l'engagement institutionnel ultérieur constitue le prédicteur le plus puissant $(\beta=0,28)$ de l'intention de persévérer et influence également les buts poursuivis $(\beta=0,16)$.

En ce qui concerne l'intégration de l'étudiant, nous pouvons observer que l'intégration sociale influence l'intégration académique $(\beta=0,21)$ et que l'intégration académique a une influence directe plus importante sur l'intention de persévérer $(\beta=0,19)$ que l'intégration sociale $(\beta=0,09)$. De manière générale, les buts poursuivis ultérieurement semblent jouer un rôle 
Figure 3. - Présentation du modèle 1

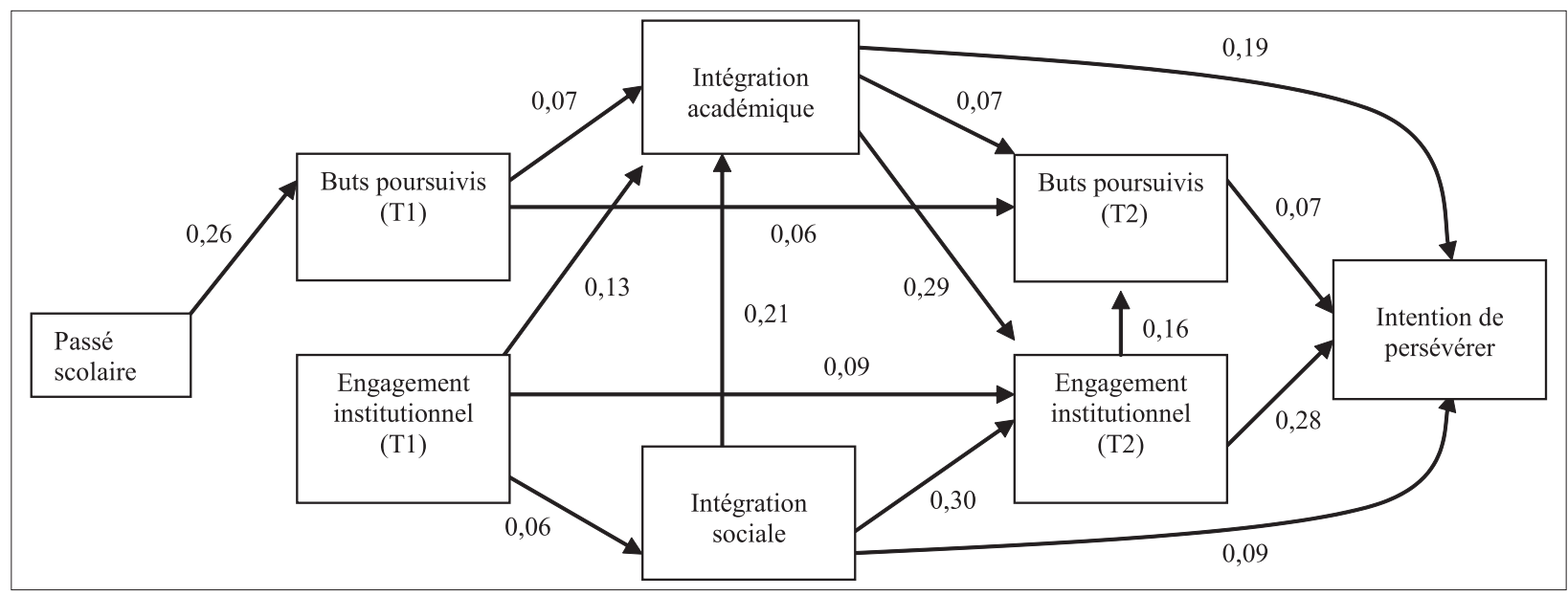

Note : T1 et T2 indiquent respectivement la première et la deuxième phases de passation du questionnaire (septembre 2005 et novembre 2005).

moins déterminant dans le processus de persévérance car ils affectent peu l'intention de persévérer $(\beta=0,07)$. II nous reste à souligner qu'une seule caractéristique individuelle du passé scolaire a un impact moins important que supposé sur les engagements initiaux, car seul le passé scolaire $(\beta=0,26)$ semble affecter les buts initialement poursuivis. Au vu de ces résultats, nous pouvons dire que seuls l'intention de persévérer $\left(R^{2}=0,21\right.$, où $R^{2}$ indique le pourcentage de variance expliqué par le modèle, voir Jöreskog \& Sörbom, 1993) et l'engagement institutionnel ultérieur $\left(R^{2}=0,23\right)$ sont prédits avec un certain succès. Les autres facteurs sont soit expliqués très faiblement (intégration académique : $R^{2}=0,05$; intégration sociale : $R^{2}=0,05$; buts poursuivis ultérieurement : $\quad R^{2}=0,07$; buts initialement poursuivis : $R^{2}=0,04$ ), soit demeurent inexpliqués (comme l'engagement institutionnel initial).

\section{Résultats du modèle 2}

Lors de cette deuxième analyse en pistes causales, nous avons introduit le sentiment d'efficacité personnelle (modèle 2) afin d'analyser son impact spécifique sur les différents facteurs clés du modèle de Tinto (1975).

Conformément aux études de Peterson (1993), il s'avère que le sentiment d'efficacité personnelle à l'arrivée à l'université (T1) influence positivement l'engagement institutionnel initial $(\beta=0,24)$ et les buts poursuivis $(\beta=0,24)$, ainsi que l'intégration académique $(\beta=0,11)$ et sociale $(\beta=0,12)$ de l'étudiant (voir la figure 4). Comme prévu, l'intégration académique $(\beta=0,23)$ et le sentiment d'efficacité personnelle initial $(\beta=0,23)$ influencent le sentiment d'efficacité personnelle ultérieur qui, à son tour, influence significativement les intentions de persévérer $(\beta=0,13)$. L'impact direct de l'intégration sociale sur le sentiment d'efficacité personnelle est faible $(\beta=0,04)$, mais une influence significative de l'intégration sociale sur l'intégration académique existe (on a toujours $\beta=0,21$ ). Il est intéressant de noter que l'inclusion du sentiment d'efficacité personnelle dans notre modèle 1 modifie certains liens existant entre les variables. En ajoutant le sentiment d'efficacité personnelle, on peut constater un relâchement de certaines des autres relations du modèle : l'impact de l'engagement institutionnel initial sur l'intégration sociale $(\beta=0,05)$, celui des buts poursuivis initialement sur l'intégration académique $(\beta=0,04)$ et celui des buts ultérieurement poursuivis sur l'intention de persévérer $(\beta=0,06)$. Nous rappelons que, quand le coefficient $\beta$ est inférieur à 0,05 , les relations ne sont pas significatives.

La comparaison des différents indices d'ajustement (voir le tableau 1) indique que le modèle 2, incluant le sentiment d'efficacité personnelle, est le modèle qui présente les meilleurs indices d'ajustement. Les modèles 1 et 2 sont significativement différents $\left(\chi^{2}(10)=41,37\right.$ à $\left.p<0,001\right)$, ce qui suggère que l'inclusion du sentiment d'efficacité personnelle enrichit effectivement le modèle de Tinto. 
Figure 4. - Présentation du modèle 2, ajoutant au modèle 1 le sentiment d'efficacité personnelle

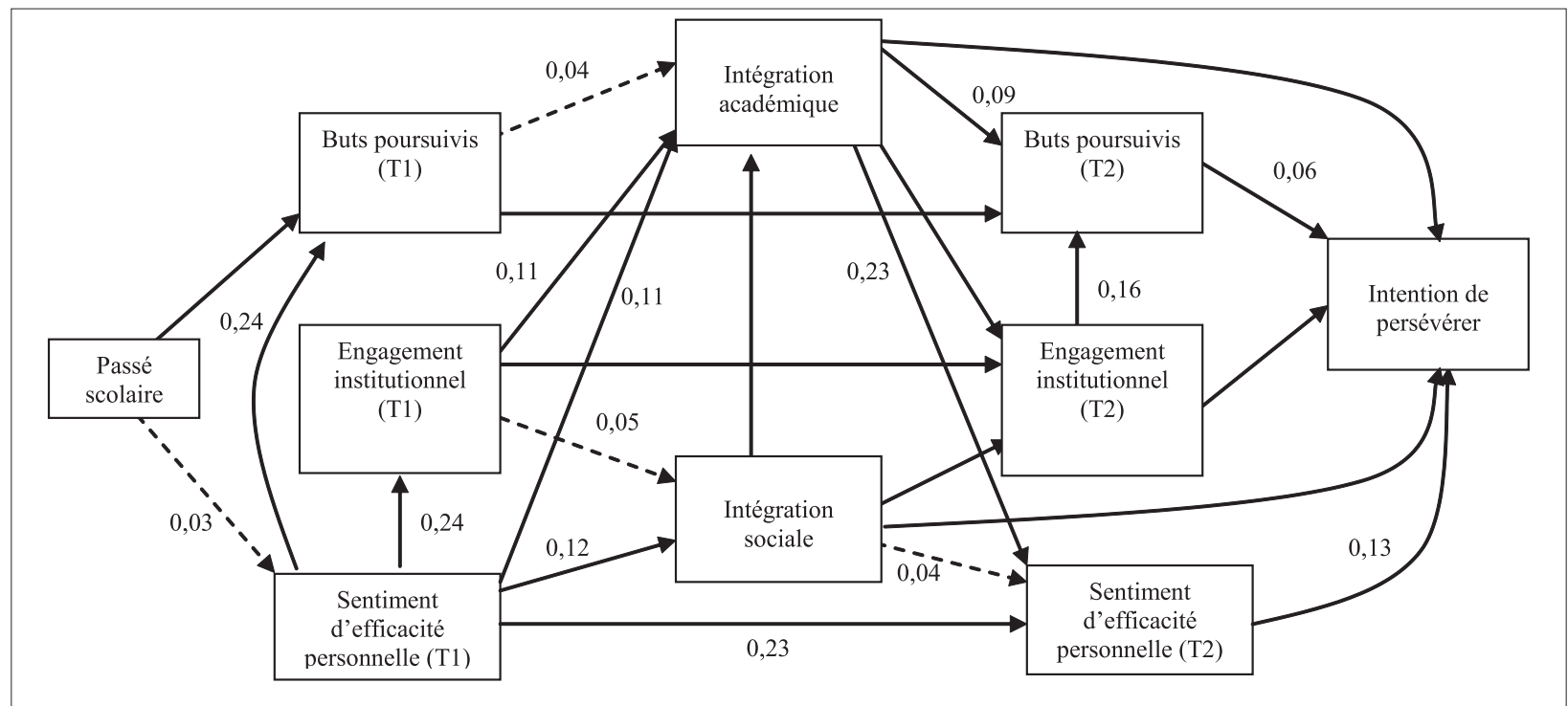

Note : les flèches pleines représentent des relations significatives ; les flèches en pointillés des relations non significatives.

Tableau 1. - Indices d'ajustement pour les deux modèles testés

\begin{tabular}{|l|c|c|c|c|c|c|c|}
\cline { 2 - 8 } \multicolumn{1}{c|}{} & ddl & $\chi^{2}$ & $\chi^{2 / d d l}$ & CFI & AGFI & sRMR & RMSEA \\
\hline Indices d'ajustement pour le modèle 1 & 11 & 51,15 & 4,7 & 0,98 & 0,98 & 0,02 & 0,04 \\
\hline Indices d'ajustement pour le modèle 2 & 21 & 92,52 & 4,4 & 0,98 & 0,98 & 0,02 & 0,04 \\
\hline
\end{tabular}

\section{DISCUSSION}

Cette étude cherchait à comparer deux modèles explicatifs de la persévérance, le premier reproduisant le modèle initial de Tinto, mais sur des données issues d'un échantillon d'étudiants belges francophones; le second intégrant les apports des théories motivationnelles au modèle éducationnel de Tinto, en y incluant notamment le sentiment d'efficacité personnelle dans la prédiction de l'intention de persévérer. Le choix d'étudier l'intention de persévérer a été justifié en raison de son lien étroit avec la persévérance effective (Bean, 1985 ; Cabrera, Nora \& Castaneda, 1993; Cabrera, Castaneda, Nora et al., 1992 ; Neuville, Frenay, Schmitz et al., 2007 ; Neuville \& Frenay, 2010) et même la réussite des étudiants (Neuville, Frenay, Schmitz et al., 2007), pour ce qu'il rend possible une détection des étudiants qui risquent d'abandonner, et également une identification aisée à l'aide des questionnaires distribués aux étudiants. Si l'on peut identifier des facteurs explicatifs de la persévérance dans les premières semaines, cela permet de mettre en œuvre à temps des actions préventives.

\section{La pertinence du modèle de Tinto}

Dans un premier temps, nous avons mis à l'épreuve le modèle de Tinto, afin de voir si les facteurs explicatifs de la persévérance avancés par cet auteur dans son student integration model (Tinto, 1975, 1987) sont pertinents dans le contexte éducatif belge francophone. Une étude exploratoire réalisée auprès de 4315 étudiants dans deux universités flamandes en Belgique a tenté de saisir les facteurs qui mènent à l'abandon en première année à l'université (Lacante, De Metsenaere, Lens et al., 2001). Sur la base du modèle de Tinto (1993), cette étude avait pu 
montrer que le parcours des étudiants au terme de la première année (abandon, échec, réussite, réussite avec grade) est lié à des différences significatives au niveau des concepts clés du modèle en question, mesurés au début de l'année académique (les étudiants ayant abandonné présentent une moins bonne intégration sociale que les étudiants ayant persisté ou échoué). En analysant systématiquement les différences entre le groupe d'étudiants ayant réussi et le groupe d'étudiants ayant échoué, les auteurs ont ainsi pu identifier les facteurs mis en jeu, sans toutefois avoir une vue globale des interactions entre ces facteurs.

La présente étude, grâce à son approche par pistes causales, permet de mesurer les interactions entre ces facteurs, et ainsi de tester certaines des hypothèses faites par Tinto dans son modèle initial (1975). La validation empirique du modèle de Tinto montre que trois facteurs semblent essentiels pour la prédiction de l'intention de persévérer: I'intégration sociale, l'intégration académique et l'engagement institutionnel. L'intégration sociale influence significativement l'intégration académique et, ensemble, elles influencent l'engagement institutionnel ultérieur, le meilleur prédicteur de l'intention de persévérer. L'impact de l'intégration sociale sur l'intégration académique et l'engagement institutionnel ultérieur est cohérent avec la littérature et les résultats de la métaanalyse de Braxton et ses collègues (1997). Même si plusieurs études affirment que l'engagement institutionnel ultérieur dépend davantage de l'intégration sociale (voir par exemple Cabrera, Nora \& Castaneda, 1993 ; Braxton, Sullivan \& Johnson, 1997), ce n'est pas le cas pour les participants de cette étude. Le rôle que joue l'engagement institutionnel ultérieur, c'est-à-dire le sentiment d'attachement envers l'institution, suggère que la perception de cet ajustement est primordiale dans la persévérance (Strauss \& Volkwein, 2004 ; Braxton, Milem \& Sullivan, 2000 ; Cabrera, Nora \& Castaneda, 1993 ; Sandler, 2000). Une mesure de cette détermination ou engagement à l'égard de son projet d'études a ainsi été validée. Bien qu'associée notamment à l'intégration académique, son impact sur l'intention de persévérer est faible, plus faible que celui de l'engagement institutionnel. Ce constat est également cohérent avec l'analyse faite par Braxton et ses collègues (1997). Cependant nous devons apporter ici une nuance. En effet, au regard de la mesure faite, nous devons rester prudents quant à ce résultat. Des travaux ultérieurs menés dans notre équipe ont en effet amélioré la mesure de ce concept (Boudrenghien, Frenay \& Bourgeois, 2009, 2010).
Une échelle de mesure de ces buts poursuivis a ainsi été validée, reprenant 15 items. Les résultats de ces études indiquent notamment le rôle clé de cet engagement pour le bien-être et la persévérance envers son but. Ils ont également montré qu'un des antécédents de l'importance accordée à s'engager vis-àvis de son projet d'études est associé à la position que ce but a par rapport aux autres buts que l'on poursuit. Ainsi plus le but que l'on poursuit eu égard à ses études est situé à un niveau élevé d'abstraction, en d'autres termes plus il fait référence à des aspects essentiels du soi, et plus il est relié à tous les autres buts que l'on poursuit (niveau d'intégration), plus son importance sera forte et renforcera l'engagement que l'on aura pour atteindre ce but, ce qui s'inscrit dans le sens du modèle de la hiérarchie des buts proposé par Carver et Scheier (1998).

La présente étude met en évidence le rôle que joue l'intégration académique dans le processus de persévérance. Contrairement aux suggestions de Braxton et ses collègues (1997) ou au regard critique de Braxton et Lien (2000) quant à la viabilité de l'intégration académique, nous pouvons constater que l'impact direct de l'intégration académique sur l'intention de persévérer est même plus important que celui de l'intégration sociale. Lorsque nous prenons en compte le sentiment d'efficacité personnelle dans nos analyses (modèle 2), nous voyons que l'intégration académique constitue une source majeure du sentiment d'efficacité personnelle mesuré durant les premières semaines à l'université. Le sentiment d'efficacité personnelle ultérieur dépend non seulement du sentiment initial, mais également de la confrontation réelle aux expériences académiques, mesurée par l'intégration académique (comme la perception des cours, la perception du soutien académique des enseignants, la perception des difficultés académiques, etc.). Ce constat suggère que cette intégration académique est importante pour soutenir le sentiment d'efficacité personnelle de l'étudiant. Nos observations sont donc cohérentes avec les suggestions de Bandura (1997) quant à l'impact de la persuasion verbale, tels les feedback fournis par l'enseignant, et celles de Eccles et Wigfield (Wigfield \& Eccles, 2000 ; Eccles \& Wigfield, 2002) suggérant que la perception de la difficulté de la tâche influence l'espérance de réussite de l'étudiant.

Qu'en est-il de l'impact de l'intégration sociale ? Le degré d'intégration sociale des étudiants joue un rôle important dans le processus de persévérance via l'influence exercée notamment sur l'intégration académique et sur l'engagement institutionnel. L'impact 
significatif de l'intégration sociale sur l'intégration académique (et non l'inverse) peut donner lieu à plusieurs explications. D'une part, il semble que plus les étudiants sont satisfaits des relations sociales avec d'autres étudiants, plus ils perçoivent les expériences académiques comme satisfaisantes (perception des cours, perception positive du souci qu'ont les enseignants à l'égard de leurs apprentissages). D'autre part, il semble que la satisfaction des besoins sociaux prime sur celle des besoins académiques durant les premières semaines. Ce constat peut être mis en lien avec le constat de Tinto (1997), selon lequel l'intégration sociale prend toute son importance durant les premières semaines, tandis que l'intégration académique et l'engagement institutionnel deviennent plus importants au cours de l'année.

Par la mise en évidence de l'importance des expériences académiques, des expériences sociales et du sentiment de congruence entre l'étudiant et l'institution choisie (mesuré par l'engagement institutionnel), nous soulignons que les modèles éducationnels constituent un cadre théorique adéquat pour étudier le processus de persévérance. Ces modèles éducationnels soulignent l'importance des interactions entre l'étudiant et l'institution et accordent en conséquence une importance cruciale au contexte institutionnel. Notre analyse partage cette philosophie en soulignant que les caractéristiques individuelles (proposées par Tinto, 1975) et les engagements initiaux sont peu déterminants dans le processus de persévérance. Seul le passé scolaire a une influence sur les buts initialement poursuivis. L'engagement institutionnel ultérieur est davantage prédit par les expériences académiques et sociales plutôt que par les engagements initiaux. Les conséquences de ce constat sont doubles. D'une part, ce constat accorde effectivement un rôle important au contexte institutionnel et permet, d'autre part, de proposer des implications pratiques pour les enseignants. Les caractéristiques d'entrée mentionnées par Tinto (1975) semblent insuffisantes pour expliquer l'introduction de l'intégration sociale, de l'intégration académique et des engagements initiaux, une des critiques effectivement adressée à son modèle (cf. Braxton, Milem \& Sullivan, 2000). Ce dernier constat souligne la nécessité de réviser le modèle de Tinto (1975) à l'aide de perspectives théoriques alternatives.

\section{Vers un modèle plus intégratif...}

La présente étude a pu montrer la supériorité du modèle combinant le modèle de Tinto et le sentiment d'efficacité personnelle. Ce constat suggère que les modèles éducationnels ont sous-estimé l'importance de certains aspects motivationnels. L'inclusion de ce sentiment d'efficacité personnelle permet d'améliorer de manière significative la prédiction de certains facteurs clés dans le processus de persévérance. En accord avec des travaux antérieurs (Peterson, 1993; Torres \& Solberg, 2001), nos résultats indiquent des effets positifs et significatifs du sentiment d'efficacité personnelle académique sur l'intégration sociale, l'intégration académique, les engagements initiaux et l'intention de persévérer. Grâce à l'impact du sentiment d'efficacité personnelle sur l'intégration sociale, la prédiction de celle-ci est améliorée. Ryan, Gheen et Midgley (1998) indiquent que les étudiants qui sont confiants dans leurs compétences participent plus facilement aux activités sociales et cherchent davantage du soutien auprès d'autres personnes. Peterson (1993) estimait que le sentiment d'efficacité personnelle était la seule caractéristique individuelle capable de contribuer significativement à l'explication de l'intégration sociale et académique. À présent, notre étude soutient cette supposition et nous avons pu constater un impact modéré du sentiment d'efficacité personnelle sur les deux types d'intégration. En indiquant un impact direct du sentiment d'efficacité personnelle ultérieur sur l'intention de persévérer, nos résultats sont en accord avec plusieurs études qui attribuent un poids prédictif plus important au sentiment d'efficacité personnelle mesuré au cours de l'année académique qu'à celui mesuré à l'entrée (Neuville, Frenay, Schmitz et al., 2007 ; Kahn \& Nauta, 2001). Les résultats présentés dans cette étude montrent que les sources majeures de ce sentiment d'efficacité personnelle ultérieur sont le sentiment mesuré à l'entrée académique et l'intégration académique.

Malgré ces constats assez convaincants et les impacts significatifs du sentiment d'efficacité personnelle sur l'intégration sociale, plaidant en faveur d'une combinaison des théories motivationnelles et éducationnelles dans l'étude de la persévérance, la prédiction de l'intégration sociale demeure faible $\left(R^{2}=0,05\right)$, mais ce n'était pas l'objectif principal de cet article de l'expliquer. Cependant, au vu de l'importance de la perception des relations sociales positives entre pairs (mesurée ici par l'intégration sociale), des recherches portant sur les sources de cette intégration sociale apparaissent nécessaires, notamment en explorant davantage les effets des expériences en classe (Tinto, 1997) et du soutien familial.

\section{Implications pratiques et perspectives}

Face à l'importance de l'intégration sociale, de l'intégration académique et de l'attachement à l'université dans la prédiction de la persévérance, la mise en 
place de programmes «de transition» (Sullivan, 1994 ; Tinto, 1997 ; Wouters, 1991) semble être un moyen efficace pour faciliter la première prise de contact avec les pairs. En proposant des discussions en groupe, des séminaires de méthode de travail, l'enseignant peut créer un climat chaleureux et propice aux interactions sociales et à l'apprentissage. Au sein de ces programmes, la promotion des interactions entre pairs et avec les enseignants constituerait un des premiers objectifs. Le recours aux pratiques pédagogiques actives ou coopératives pourrait également avoir un effet bénéfique sur le développement cognitif et social de l'étudiant.

II existe différentes stratégies pour soutenir et améliorer le sentiment d'efficacité personnelle. Parmi les sources du sentiment d'efficacité personnelle se trouve d'abord la persuasion verbale (Bandura, 1997). En utilisant des feedback positifs et encourageants (comme justement la persuasion verbale), les enseignants pourraient davantage agir sur la perception qu'ont les étudiants de leurs compétences (Galand \& Vanlede, 2004 ; Vanlede, Bourgeois, Galand et al., 2009). Vanlede et ses collègues (2009) soulignent que les souvenirs liés aux performances passées constituent une autre source d'influence du sentiment d'efficacité personnelle. Comme les souvenirs d'échec diminuent le sentiment d'efficacité personnelle alors que les souvenirs de réussite le renforcent, des interventions destinées à modifier la spécificité des souvenirs ont mis en évidence la possibilité d'améliorer le sentiment d'efficacité personnelle.

Deux pistes nous semblent devoir être mises en évidence pour mieux cerner cette dimension d'engagement vis-à-vis des buts poursuivis par un étudiant. D'une part, la mesure de ce concept devrait être améliorée afin d'évaluer plus précisément le degré auquel l'étudiant est déterminé à atteindre son but ainsi que sa volonté à faire des efforts pour l'atteindre (Affleck, Tennen, Zautra et al., 2001 ; Blustein, Ellis \& Devenis, 1989 ; Brunstein, 1993 ; Brunstein, Schultheiss \& Grässmann, 1998). La mesure plus précise de cet engagement permettrait peut-être de mieux mettre en évidence son impact sur l'intention de persévérance. D'autre part, les résultats de la présente étude soulignent le peu d'éléments dont nous disposons pour expliquer cette détermination à poursuivre son projet d'études. Les travaux de Boudrenghien, Frenay et Bourgeois (2009) tentent de répondre à cette limite en étudiant les antécédents de cet engagement. Ils mettent ainsi en évidence l'impact de la représentation que l'on se fait de son but en termes de niveau d'abstraction (être un certain type de personne versus faire un certain type d'action) et de degré d'intégration (degré avec lequel on perçoit que ce but contribue aux autres buts que l'on poursuit dans sa vie). La place qu'occupe son projet d'études au sein de cette structure hiérarchique de ses buts ainsi représentée influence l'importance que l'on accorde à ce projet, ainsi que le sentiment que l'on pourra l'atteindre. Ceci renforcera le degré de détermination que l'on mettra pour atteindre ce but, l'engagement envers ce projet. Accompagner les étudiants à prendre conscience des buts qu'ils poursuivent, à les positionner les uns par rapport aux autres, à les articuler peut ainsi les amener à travailler sur leur engagement ou détermination à l'entrée à l'université.

La présente étude donne également des pistes prometteuses pour des recherches futures. II pourrait être en effet intéressant de rechercher si le modèle de Tinto, complété par l'apport des modèles motivationnels (modèle 2), peut être efficace dans la prédiction de la persévérance effective et de la performance académique. Neuville et ses collègues (2007) ont constaté un impact direct des intentions de persévérer sur la performance des étudiants à la session d'examen de janvier $(\beta=0,09)$. Les croyances d'efficacité personnelle mesurées au cours de l'année influencent également la performance (Multon, Brown \& Lent, 1991; Kahn \& Nauta, 2001; Gore, Leuwerke \& Turley, 2006). Dans une étude récente (Neuville \& Frenay, 2010), les auteurs soulignent que l'intention de persévérer et le sentiment d'efficacité personnelle mesurés au cours de l'année prédisent au mieux la persévérance effective.

Dans les limites rencontrées par le modèle de Tinto (1975) pour expliquer l'intégration sociale, trois perspectives s'ouvrent. Premièrement, l'intégration sociale n'a été mesurée que par la satisfaction relative des étudiants dans les relations avec les pairs, qui est une seule facette de l'intégration sociale définie par Tinto (1975). L'utilisation d'une échelle plus complète pourrait " capturer » le concept dans sa globalité et permettre ainsi de réévaluer l'efficacité de sa prédiction au sein du modèle de Tinto. Deuxièmement, une mise à l'épreuve du modèle révisé par Tinto en 1997 peut dessiner une autre perspective. Dans cette révision de son modèle, il accorde aux expériences sociales en classe (comme la collaboration entre étudiants) un rôle prédicteur important de l'intégration sociale et de l'intégration académique. Selon Tinto, la classe ou l'amphithéâtre est un lieu de rencontre, entre étudiants et avec l'enseignant, d'où peut émerger l'intégration sociale. En favorisant les interactions sociales 
dans le travail intellectuel (notamment via des travaux coopératifs), les étudiants peuvent trouver des réponses simultanément aux besoins académiques et sociaux, sans devoir sacrifier l'un au bénéfice de l'autre.

Julia Schmitz

julia.schmitz@uclouvain.be

Groupe interdisciplinaire de recherche sur

la socialisation, l'éducation et la formation, Université catholique de Louvain, Belgique

Mariane Frenay

GIRSEF, Chaire UNESCO de pédagogie universitaire, Université catholique de Louvain, Belgique

Sandrine Neuville

Groupe interdisciplinaire de recherche sur la socialisation, l'éducation et la formation, Université catholique de Louvain, Belgique
Gentiane Boudrenghien

Groupe interdisciplinaire de recherche sur la socialisation, l'éducation et la formation, Université catholique de Louvain, Belgique

Vincent Wertz

Technologies de l'information et de la communication, électronique et mathématiques appliquées, Université catholique de Louvain, Belgique

Bernadette Noël

Département de sciences politiques, sociales et de communication, facultés universitaires catholiques de Mons, Belgique

Jacquelynne Eccles

Institute of social research, University of Michigan, Ann Harbor, États-Unis

\section{NOTES}

(1) Dans le système éducatif de la Communauté française de Belgique, il n'existe pas de système d'évaluation communautaire délivrant ce titre, comme le baccalauréat français. Le CESS est donc attribué sur la base des résultats obtenus par les élèves aux cours suivis durant leur sixième année.

(2) Ce concept de "goal commitment " de Tinto correspond à l'effort déployé pour atteindre ses buts personnels et réfère, dans son modèle, à l'effort déployé pour obtenir un diplôme.

(3) Student attrition model est le nom original de ce modèle.

(4) Ici, il s'agit d'étudiants dits "traditionnels ", c'est-à-dire d'étudiants entrant à l'université pour la première fois, âgés de moins de 24 ans, non mariés et n'appartenant pas à une population minoritaire.

(5) Dans cette étude, les chercheurs ont testé plusieurs modèles alternatifs et le modèle présentant la meilleure adéquation (cf modèle en pistes causales) aux données recueillies constitue un modèle incluant la relation entre soutien familial et des proches et l'intégration sociale, même si au départ, elle n'était pas postulée dans leur modèle initial.
(6) Cette théorie a élargi la théorie sociale cognitive de Bandura en l'appliquant au domaine spécifique des choix d'orientation d'études et des choix professionnels.

(7) L'alpha de Cronbach constitue un indice de consistance interne de l'échelle, indiquant le degré auquel l'ensemble des items utilisés pour mesurer une variable mesurent bien la même chose. Cet indice varie entre 0 et 1 , et les valeurs au-delà de 0,70 indiquent une consistance interne élevée, voire très élevée.

(8) La réussite à la fin de la première année de l'université est jugée sur la base d'une moyenne générale de tous les cours de l'année présentés et d'une mention attribuée par le jury de l'année. La réussite n'est acquise que si les étudiants ont un minimum de $60 \%$ de moyenne générale, sans avoir une seule note inférieure à la moyenne. Ces grades, ou mentions, de satisfaction, distinction, grande distinction et plus grande distinction correspondent à une moyenne générale égale ou supérieure respectivement à $60 \%, 70 \%, 80 \%$ et $90 \%$.

(9) Ce résultat est d'autant plus surprenant que nous avions montré que le diplôme de la mère a par contre un impact important sur la performance des étudiants, même si les autres variables sont contrôlées (Neuville, Frenay, Schmitz et al., 2007).

\section{BIBLIOGRAPHIE}

AFFLECK G., TENNEN H., ZAUTRA A., URROWS S. ABELES M. \& KAROLY P. (2001). « Women's pursuit of personal goals in daily life with fibromyalgia: A valueexpectancy analysis". Journal of Consulting and Clinical Psychology, vol. 69, $n^{\circ} 4$, p. 587-596.

BANDURA A. (1986). " The explanatory and predictive scope of self-efficacy theory ". Journal of Clinical and Social Psychology, vol. 4, n 3, p. 359-373.

BANDURA A. (1997). Self-efficacy: The exercise of control. New York: W.H. Freeman.

BEAN J. (1980). « Dropouts and turnover: The synthesis and test of a causal model of student attrition ». Research in Higher Education, vol. 12, n² 2, p. 155-187.
BEAN J. (1983). "The application of a model of turnover in work organizations to the student attrition process ". Review of Higher Education, vol. 6, $\mathrm{n}^{\circ} 2$, p. $129-148$.

BEAN J. (1985). « Interaction effects based on class level in an explanatory model of college student dropout syndrome ". American Educational Research Journal, vol. $22, n^{\circ} 1$, p. 35-64.

BEAN J. \& EATON S. (2000). « A psychological model of college student retention ». In J. Braxton (dir.), Reworking the student departure puzzle. Nashville: Vanderbilt University Press. 
BEAN J. \& METZNER B. (1985). «A conceptual model of nontraditional undergraduate student attrition ". Review of Educational Research, vol. 55, $\mathrm{n}^{\circ} 4$, p. 485-540.

BEAN J. \& VESPER N. (1990). "Quantitative approaches to grounding theory in data: Using Lisrel to develop a local model and theory of student attrition ". Communication présentée à l'Annual meeting of the American educational research association, Boston.

BENTEIN K., FRENAY M., VERWAERDE A., BOURGEOIS É. \& GALAND B. (2003). "Understanding academic failure among the first entrance university students for promoting academic achievement ". Communication présentée à la Biennal conference of the European association for research on learning and instruction, Padoue, Italie.

BERGER J. \& MILEM J. (1999). "The role of student involvment and perceptions of integration in a causal model of student persistence ". Research in Higher Education, vol. $40, n^{\circ} 6$, p. 641-664.

BERNIER A., LAROSE S. \& WHIPPLE N. (2005). « Leaving home for college: A potentially stressful event for adolescents with preoccupied attachment patterns". Attachment \& Human Development, vol. $7, \mathrm{n}^{\circ}$ 2, p. 171 185.

BETZ N. \& HACKETT G. (1981). «The relationship of careerrelated self-efficacy expectation to perceived career options in college women and men ". Journal of Counseling Psychology, vol. 28, $\mathrm{n}^{\circ}$ 5, p. 399-410.

BLUSTEIN D., ELLIS M. \& DEVENIS L. (1989). «The development and validation of a two-dimensional model of the commitment to career choices process ". Journal of Educational Behavior, vol. 35, $\mathrm{n}^{\circ}$ 3, p. 342-378.

BONG M. (2001). « Between-and within-domain relations of academic motivation among middle and high school students: Self-efficacy, task value, and achievement goals". Journal of Educational Psychology, vol. 93 $\mathrm{n}^{\circ}$ 1, p. 23-34.

BOUDRENGHIEN G., FRENAY M. \& BOURGEOIS É. (2009). "La transition de l'enseignement secondaire vers l'enseignement supérieur. Antécédents de l'engagement envers son but de formation ". Les cahiers de recherche en éducation et formation, $\mathrm{n}^{\circ} 70$.

BOUDRENGHIEN G., FRENAY M. \& BOURGEOIS É. (2010). «La transition de l'enseignement secondaire vers l'enseignement supérieur ». In S. Neuville, M. Frenay, B. Noël \& V. Wertz (dir.), Persévérer et réussir à l'Université [sous presse]. Louvain-la-Neuve : Presses universitaires de Louvain.

BRAXTON J. (2000). Reworking the student departure puzzle. Nashville: Vanderbilt University Press.

BRAXTON J. \& HIRSCHY A. (2005). «Theoretical developments in college student departure ». In A. Seidman (dir.), College student retention: Formula for student success. Westport: Greenwood Press, p. 61-87.

BRAXTON J. \& LIEN L. (2000). «The viability of academic integration as a central construct in Tinto's interactionalist theory of college student departure ». In J. Braxton (dir.), Reworking the student departure puzzle. Nashville : Vanderbilt University Press.

BRAXTON J., MILEM J. \& SULLIVAN A. (2000). «The influence of active learning on the college student departure process: Toward a revision of Tinto's theory ". Journal of Higher Education, vol. 75, n 5, p. 569-590.
BRAXTON J., SULLIVAN A. \& JOHNSON R. (1997). "Appraising Tinto's theory of college student departure ». In J. Smart (dir.), Higher education: Handbook of theory and research, vol. 12. New York: Agathon Press, p. 107-164.

BRUNSTEIN J. (1993). «Personal goals and subjective wellbeing: A longitudinal study ». Journal of Personality and Social Psychology, vol. 65, n 5, p. 1061-1070.

BRUNSTEIN J., SCHULTHEISS O. \& GRÄSSMANN R. (1998). "Personal goals and emotional well-being: The moderating role of motive dispositions ". Journal of Personality and Social Psychology, vol. 75, $\mathrm{n}^{\circ} 2$, p. 494-508.

BYRNE B. (1998). Structural equation modeling with Lisrel, Prelis, and Simplis: Basic concepts, applications, and programming. Mahwah : Lawrence Erlbaum.

CABRERA A., NORA A. \& CASTANEDA M. (1993). "College persistence: Structural equations modeling test of an integrated model of student retention ». The Journal of Higher Education, vol. 64, $\mathrm{n}^{\circ} 2$, p. 123-139.

CABRERA A., CASTANEDA M., NORA A. \& HENGSTLER D. (1992). "The convergence between two theories of college persistence ». The Journal of Higher Education, vol. $63, n^{\circ} 2$, p. $143-164$

CARVER C. \& SCHEIER M. (1998). On the self-regulation of behavior. Cambridge : Cambridge university Press.

CHEMERS M., HU L. \& GARCIA B. (2001). «Academic selfefficacy and first-year college student performance and adjustment ». Journal of Educational Psychology, vol. 93, $\mathrm{n}^{\circ} 1$, p. 55-64.

COOKE R., BEWICK B., BARKHAM M., BRADLEY $M$. \& AUDIN K. (2006). "Measuring, monitoring and managing the psychological well-being of first year university students ". British Journal of Guidance \& Counselling, vol. 34, $n^{\circ} 4$, p. 505-517.

ECCLES J. \& WIGFIELD A. (2002). " Motivational beliefs, values, and goals". Annual Review of Psychology, vol. $53, n^{\circ} 1$, p. 109-132.

GALAND B. \& FRENAY M. (2005). L'approche par problèmes et par projets dans l'enseignement supérieur. Impact, enjeux et défis. Louvain-la-Neuve : Presses universitaires de Louvain.

GALAND B. \& VANLEDE M. (2004). « Le sentiment d'efficacité personnelle dans l'apprentissage et la formation. Quel rôle joue-t-il ? D'où vient-il ? Comment intervenir ? "Les cahiers de recherche en éducation et formation, $\mathrm{n}^{\circ} 29$.

GORE P., LEUWERKE W. \& TURLEY S. (2006). «A psychometric study of the college self-efficacy inventory ". Journal of College Student Retention: Research, Theory and Practice, vol. $7, \mathrm{n}^{\circ} 3$, p. 227-244.

HU L. \& BENTLER P. (1998). « Fit indices in covariance structure modeling: Sensitivity to underparameterized model misspecification ». Psychological Methods, vol. 3, $\mathrm{n}^{\circ} 4$, p. $424-453$

JÖRESKOG K. \& SÖRBOM D. (1993). LISREL 8: Structural equation modeling with the SIMPLIS command language. Chicago : Scientific software international.

KAHN J. \& NAUTA M. (2001). "Social-cognitive predictors of first-year college persistence: The importance of proximal assessment ». Research in Higher Education, vol. $42, n^{\circ} 6$, p. 633-652. 
KARPANTY R. (1998). Role of communication apprehension, college self-efficacy, peer integration and faculty integration on college persistence. Thèse de doctorat, communication, University of Wisconsin, Milwaukee.

LACANTE M., DE METSENAERE M., LENS W., VAN ESBROECK R. et al. (2001). " Drop-out in het hoger onderwijs: Onderzoek naar achtergronden en motieven van drop-out in het eerste jaar hoger onderwijs " (Eindrapport OBPWO $\mathrm{n}^{\circ}$ 98.11). Louvain: Katholieke Universiteit Leuven et Bruxelles: Vrije Universiteit Brussel.

LENT R., BROWN S. \& HACKETT G. (1994). « Toward a unifying social cognitive theory of career and academic interest, choice, and performance ". Journal of Vocational Behavior, vol. 45, n 1, p. 79-122.

MANNAN M. (2001). " An assessment of the academic and social integration as perceived by the students in the University of Papua New Guinea ». Higher Education, vol. $41, n^{\circ} 3$, p. 283-298.

McCUBBIN I. (2003). «An examination of criticisms made of Tinto's 1975 student integration model of attrition ". Rapport de recherche. Disponible sur Internet à l'adresse : <http://www.psy.gla.ac.uk/ steve/localed/ icubb.pdf> (consulté le 13 octobre 2010).

MEYERS L., GAMST G. \& GUARINO A. (2006). Applied multivariate research: Design and interpretation. Thousand Oaks : Sage.

MULTON K., BROWN S. \& LENT R. (1991). « Relation of selfefficacy beliefs to academic outcomes: A meta-analytic investigation". Journal of Counseling Psychology, vol. $38, n^{\circ} 1$, p. 30-38.

NEUVILLE S. \& FRENAY M. (2010). «La persévérance des étudiants de $1^{\mathrm{er}}$ baccalauréat à la lumière du modèle expectancy-value ». In C. Michaut \& M. Romainville (dir.), Réussite, échec et abandon dans l'enseignement supérieur [sous presse]. Bruxelles: De Boeck.

NEUVILLE S., FRENAY M., SCHMITZ J., BOUDRENGHIEN G. NOËL B. \& WERTZ V. (2007). "Tinto's theoretical perspective and expectancy-value paradigm: A confrontation to explain freshmen academic achievement ». Psychologica Belgica, vol. 47, n¹-2, p. 31-50.

OSWALD D. \& CLARK E. (2003). «Best friends forever?: High school best friendships and the transition to college ». Personal Relationships, vol. 10, $\mathrm{n}^{\circ} 2$, p. 187196.

PARKER J., SUMMERFELD L., HOGAN M. \& MAJESKI S. (2004). "Emotional intelligence and academic success: Examining the transition from high school to university ». Personality and Individual Differences, vol. 36, $\mathrm{n}^{\circ} 1$, p. 163-172.

PARMENTIER P. (1994). La réussite des études universitaires: facteurs structurels et processuels de la performance académique en première année en médecine. Thèse de doctorat, sciences psychologiques et sciences de l'éducation, Université catholique de Louvain, Louvain-la-Neuve, Belgique.

PASCARELLA E. \& CHAPMAN D. (1983). «A multiinstitutional, path analytic validation of Tinto's model of college withdrawal ». American Educational Research Journal, vol. 20, $\mathrm{n}^{\circ} 1$, p. 87-102.

PASCARELLA E. \& TERENZINI P. (1979). «Interaction effects in Spady's and Tinto's conceptual models of college dropout ». Sociology of Education, vol. 52, n 4, p. 197210.

PASCARELLA E. \& TERENZINI P. (1980). «Predicting freshman persistence and voluntary dropout decisions from a theoretical perspective ". Journal of Higher Education, vol. 51, $\mathrm{n}^{\circ} 1$, p. 60-75.

PASCARELLA E. \& TERENZINI P. (1983). «Predicting voluntary freshman year persistence/withdrawal behavior in a residential university: $A$ path analytic validation of Tinto's model ». Journal of Educational Psychology, vol. $75, n^{\circ} 2$, p. $215-222$.

PASCARELLA E. \& TERENZINI P. (1991). "How college affects students ". San Francisco : Jossey-Bass.

PASCARELLA E. \& TERENZINI P. (2005). How college affects students: A third decade of research, vol. 2. San Francisco : Jossey-Bass.

PASCARELLA E., DUBY P. \& IVERSON B. (1983). «A text and reconceptualization of a theoretical model of college withdrawal in a commuter institution setting". Sociology of Education, vol. 56, $\mathrm{n}^{\circ} 2$, p. 88-100.

PAUL E. \& BRIER S. (2001). "Friendsickness in the transition to college: Precollege predictors and college adjustment correlates ". Journal of Counseling \& Development, vol. 79, $\mathrm{n}^{\circ} 1$, p. 77-89.

PETERSON S. (1993). «Career decision-making self-efficacy and institutional integration of underprepared college students ". Research in Higher Education, vol. 34, $n^{\circ} 6$, p. 659-685.

ROBBINS S., LAUVER K., LE H., DAVIS D., LANGLEY R. \& CARLSTROM A. (2004). «Do psychosocial and study skill factors predict college outcomes? A metaanalysis ». Psychological Bulletin, vol. 130, $\mathrm{n}^{\circ}$ 2, p. 261288.

ROMAINVILLE M. (2000). L'échec dans l'université de masse. Paris : L'Harmattan.

ROMAINVILLE M. (2005). "Quelques interrogations sur l'échec à |'Université ". Les cahiers de recherche en éducation et formation, $\mathrm{n}^{\circ} 39, \mathrm{p} .18-22$.

RYAN A., GHEEN M. \& MIDGLEY C. (1998). « Why do some students avoid asking for help? An examination of the interplay among students' academic efficacy, teachers' social emotional role, and the classroom goal structure ». Journal of Educational Psychology, vol. 90, $\mathrm{n}^{\circ} 3$, p. 528-535.

SANDLER M. (2000). " Career decision-making self-efficacy, perceived stress, and an integrated model of student persistence: A structural model of finances, attitudes, behavior, and career development ". Research in Higher Education, vol. 41, n 5, p. 537-580.

SCHMITZ J. (2009). La persévérance en première année à l'université : sources et effets de l'intégration sociale. Thèse de doctorat, sciences psychologiques et de l'éducation, Université catholique de Louvain, Louvain-la-Neuve, Belgique. Disponible sur Internet à l'adresse : <http://hdl. handle.net/2078.1/29003> (consulté le 13 octobre 2010).

SCHMITZ J., FRENAY M., NEUVILLE S., BOUDRENGHIEN G., NOËL B. \& WERTZ V. (2007). "The role of social integration and peer relationships in the persistence of firstyear students at university: Analysis of determinants and effects ". Communication présentée à la 12th Biennal conference for research on learning and instruction, EARLI, Budapest. 
SHAVER P., FURMAN W. \& BUHRMESTER D. (1985). «Transition to college: Network changes, social skills, and loneliness ». In S. Duck \& D. Perlman (dir.), Understanding personal relationships: An interdisciplinary approach. Londres : Sage, p. 193-219.

SOLBERG V., O'BRIEN K., VILLARREAL P. et al. (1993). "Self-efficacy and Hispanic college students: Validation of the college self-efficacy inventory ". Hispanic Journal of the Behavioral Sciences, vol. 15, $\mathrm{n}^{\circ} 1$, p. 80-95.

SPADY W. (1970). "Dropouts from higher education: An interdisciplinary review and synthesis ». Interchange, vol. $1, \mathrm{n}^{\circ} 1$, p. 64-85.

STRAUSS L. \& VOLKWEIN J. (2004). «Predictors of student commitment at two-year and four-year institutions". The Journal of Higher Education, vol. 75, n 2, p. 203227.

SULLIVAN C. (1994). "Creating a supportive environment for college freshmen: Underlying dimensions of the freshmen interest group program ». Innovative Higher Education, vol. 19, $\mathrm{n}^{\circ}$ 2, p. 83-97.

TINTO V. (1975). «Dropout from higher education: A theoretical synthesis of recent research ". Review of Educational Research, vol. 45, $n^{\circ} 1$, p. 89-125.
TINTO V. (1987). Leaving college: Rethinking the causes and cures of student attrition. Chicago : University of Chicago Press.

TINTO V. (1993). Leaving college: Rethinking the causes and cures of student attrition [2 ${ }^{\mathrm{e}}$ ed.]. Chicago : University of Chicago Press.

TINTO V. (1997). "Classrooms as communities: Exploring the educational character of student persistence ". Journal of Higher Education, vol. 68, n 6, p. 599-622.

TORRES J. \& SOLBERG S. (2001). «Role of self-efficacy, stress, social integration, and family support in latino college student persistence and health ". Journal of Vocational Behavior, vol. 59, $n^{\circ} 1$, p. 53-63.

VANLEDE M., BOURGEOIS É., GALAND B. \& PHILIPPOT P. (2009). "Sources of academic self-efficacy-beliefs: The role of the specificity level of autobiographical memories about academic performance". Les cahiers de recherche en éducation et formation, $\mathrm{n}^{\circ} 67, \mathrm{p} .1-11$.

WIGFIELD A. \& ECCLES J. (2000). «Expectancy-value theory of achievement motivation ". Contemporary Educational Psychology, vol. 25, $\mathrm{n}^{\circ} 1$, p. 68-81.

WOUTERS P. (1991). Les cours d'été à l'UCL. Un dispositif de pédagogie universitaire en vue de faciliter la transition entre l'enseignement secondaire et l'université. Louvain-la-Neuve : Université catholique de Louvain. 